\title{
Alkali cluster films on insulating substrates: comparison between scanning force microscopy and extinction data
}

\author{
F. Balzer ${ }^{1}$, S.D. Jett ${ }^{2}$, H.-G. Rubahn ${ }^{3}$ \\ Max-Planck-Institut für Strömungsforschung, Bunsenstr.10, D-37073 Göttingen, Germany \\ Abteilung Molekulare Biologie, Max-Planck-Institut für Biophysikalische Chemie, Am Faßberg 11, D-37077 Göttingen, Germany
}

Received 10 September 1998

\begin{abstract}
Extinction spectra of alkali cluster films on mica substrates are recorded as a function of alkali metal deposition time. They are reproduced quantitatively by theoretical spectra of oblate spheroids, using cluster number density $n$, average cluster semi-axis $\langle a\rangle$ parallel to the mica surface, and mean ellipticity $\left\langle R_{\perp}\right\rangle$ perpendicular to the surface obtained from scanning force microscopy images. It is found that reliable values of average cluster semi-axes can be obtained from optical spectra only by taking the distribution of perpendicular-plane ellipticities into account. (C) 1998 Elsevier Science B.V. All rights reserved.
\end{abstract}

\section{Introduction}

Large alkali clusters, grown on insulating substrates, are interesting objects for fundamental and applied studies due to their extraordinary optical properties and their electronic simplicity [1]. Light absorption in those clusters results in a collective electronic excitation, which shows, as a function of cluster size, shape and exciting wavelength, a resonant behaviour [2]. At the spectral position of those 'surface plasmon' resonances the electromagnetic fields at the cluster surfaces are strongly enhanced.

\footnotetext{
${ }^{1}$ Present address: Department of Chemistry, Stanford University, Stanford, CA 94305-5080, USA.

${ }^{2}$ Present address: Department of Cell Biology and Physiology, University of New Mexico School of Medicine, Albuquerque, NM 87131, USA.

${ }^{3}$ Corresponding author. E-mail: hrubahn@gwdg.de
}

This field enhancement effect makes second- (viz. second-harmonic generation (SHG)) [3] and higherorder (viz. degenerate four-wave mixing) [4] non-linear optical processes easily detectable. On the other hand, by taking advantage of the strong SHG in the clusters and by using ultrashort pulse lasers, the dynamics of the dephasing of the initial collective excitation (i.e., the decay of the surface plasmon excitation) could be investigated as a function of mean cluster size [5]. The subsequent electron-phonon and phonon-phonon coupling [6,7], as well as the final bond-breaking mechanism [8], were also subject of successful studies.

In contrast to free metal clusters, the investigation of surface-bound clusters, grown from adsorbed atoms, has significant experimental advantages: (1) their temperature is well known; (2) their number density is high $\left(10^{8}-10^{12} \mathrm{~cm}^{-2}\right)$ and can be varied by changing surface temperature and flux of the 
impinging atoms [9]; (3) the symmetry break at the supporting surface makes surface SHG processes possible even for clusters much smaller than the wavelength of the exciting light; and (4) their average size and shape can be easily changed just by varying the amount of atoms, initially deposited on the surface. However, a closer look shows that especially the latter point, namely the distribution of cluster sizes and shapes at the surface, imposes many problems and uncertainties on the experiment.

For example, the growth of noble metal clusters and rough films on insulating substrates [10], as well as the growth of alkali films [11], have been investigated for about 30 years, initially by transmission electron microscopy but more recently with scanning probe microscopies [12,13]. In the course of those experiments it has been found that the size distribution of the clusters on the surface usually can be well described by a double-Gaussian distribution around a mean cluster size [14] as long as coalescence of the clusters does not occur. The double-Gaussian distribution describes a large density of small clusters, grown independently of each other, in contrast to a log-normal distribution, which describes an enhanced density of larger clusters due to coagulation.

This finding has been applied later to large alkali (sodium) clusters on lithium fluoride substrates [15] in order to fit measured linear extinction spectra to spectra obtained from a Mie scattering calculation [16]. Although the measurement of linear extinction spectra is an experimental method that can be implemented easily in situ, the interpretation in terms of cluster size distributions requires a more sophisticated experimental approach. In particular, one has to take into account that the clusters are non-spherical at the surface, implying, for example, that a T-matrix approach has to be introduced as a generalization of the classical Mie scattering [17,18]. Even then, the numerous fitting parameters necessary for dealing appropriately with (i) the absolute value of extinction (i.e., number density of clusters) and (ii) the depolarization, retardation and interaction-induced shifts and broadenings of the surface plasmon peaks (i.e., parameters concerning shape and size distributions of the clusters and the optical properties of the substrate) do not allow a unique description of the clusters that lead to the measured extinction spectrum.
In our present research we have followed two routes for solving this problem. The first route, the results of which are presented in this Letter, compares theoretical extinction spectra, deduced from morphological information that has been obtained by direct nanoprobing of the clusters with an scanning force microscope (SFM) with measured extinction spectra. Recently the usefulness of a direct comparison between SFM information and spectroscopic ellipsometric data in terms of morphological information has been demonstrated for thin Ag films [19] and for $\mathrm{Ge}$ islands grown on $\mathrm{Si}(100)$ [20]. Our present Letter goes a significant step further in that completely predicted extinction spectra are compared with measured ones.

The second strategy has involved the study of the microscopic growth process of the cluster films in more detail by applying atomic beam diffraction methods such as helium atom scattering. The corresponding results will be published in the near future [21].

\section{Experimental}

Sodium cluster size distributions have been generated on air-cleaved and outgassed muscovite mica samples (grade V4, Structure Probe, Inc., West Chester, PA) by ultrahigh vacuum (UHV) evaporation of sodium $(\mathrm{Na})$ atoms at room temperature from an alkali dispenser (SAES Getters). At the surface the atoms migrate to defect sites where they stick and start forming large alkali clusters. In principle, this should result in a cluster density approximately equal to the defect density of the surface. However, more accurate measurements have shown that in the initial stage of the process the cluster density increases up to a limiting value, which depends on the diffusion energy of the atoms on the surface and on the flux of the impinging atoms. This is followed by coagulation processes $[14,22]$. During all these stages of growth the mean cluster size increases. Hence, for a theoretical reproduction of measured extinction spectra the cluster density is, within certain ranges, a free fitting parameter. Neglecting this and simply assuming that the cluster density equals the defect density might lead to wrong values of mean cluster sizes. 
The flux of $\mathrm{Na}$ atoms approaching the sample has been controlled in our experiment by a hot wire (Langmuir Taylor) detector and is of the order of $10^{12}$ atoms $/\left(\mathrm{cm}^{2} \mathrm{~s}\right)$. Linear extinction spectra [23] have been taken in the process of $\mathrm{Na}$ evaporation by use of a collimated light beam (1 mm diameter) from a $50 \mathrm{~W}$ halogen lamp and a spectrometer (Ocean Optics). For sodium, the dipole Mie resonance for small spheres is located at $\lambda \approx 377 \mathrm{~nm}$ [2]; therefore, extinction spectra in the visible between $\lambda=350$ and $\lambda=750 \mathrm{~nm}$ were taken. In addition to the spectra obtained under normal incidence, spectra taken at finite angles and employing s- and ppolarized light were used to probe the shape of the clusters. This method takes advantage of the possible excitation of dipole plasmon oscillations along the half axis perpendicular to the substrate surface for p-polarized light, which is not allowed for s-polarized light or under normal incidence. The spectra have been calibrated initially with the help of reference spectra, which included the spectral response of the lamp, the spectrometer and the uncovered mica surface for the implemented polarizations and angles of incidence. Since mica absorbs light efficiently below $320 \mathrm{~nm}$ the UV part of the spectrum could not be observed. After finishing the cluster growth process, control measurements ensured that the extinction spectra of the sample in UHV did not change for at least several hours.

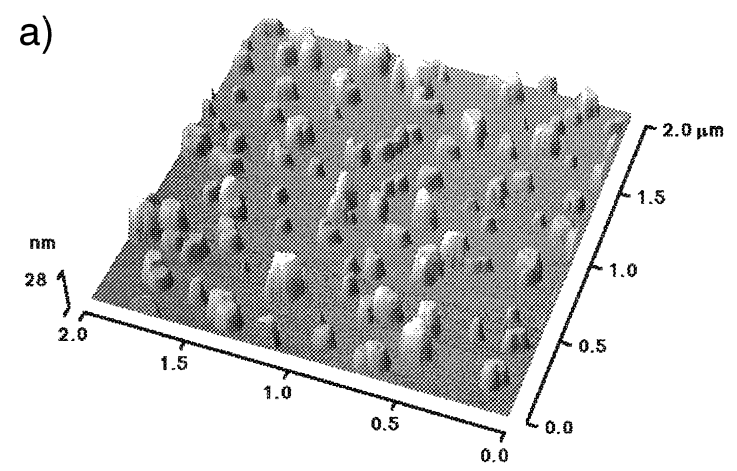

Using a magnetic transfer system, the sample was removed from the UHV chamber into a transfer chamber, where it was exposed to ambient air. Measurements were performed using a scanning force microscope (Nanoscope IIIa, Digital Instruments, Santa Barbara, CA) under ambient air conditions $\left(18-25^{\circ} \mathrm{C}, 15-50 \%\right.$ relative humidity). Scanning typically took place $1 \mathrm{~h}$ after sample transfer out of the UHV. We used silicon cantilevers with a $2 \mathrm{~N} / \mathrm{m}$ force constant, $160 \mathrm{kHz}$ resonant frequency, and 10 nm average tip radius (Ultralevers, Park Scientific, Sunnyvale, CA). Images were normally collected in tapping mode, at $2-4 \mathrm{~Hz}$ scan frequency.

\section{Results and discussion}

Fig. 1a is a $2 \times 2 \mu \mathrm{m}^{2}$ SFM image of a distribution of Na-like (probably $\mathrm{NaOH}$, see below) clusters on mica, grown for $750 \mathrm{~s}$ at a surface temperature of $T_{\mathrm{S}}=300 \mathrm{~K}$ on cleaved mica. The height scale is given on the left-hand-side. As seen in Fig. 1b, which is a scan through two clusters on the surface, the clusters are very flat with ratios of small semi-axis (a) parallel to the surface to semi-axis $(c)$ perpendicular to the surface, $R_{\perp}=c / a$, of the order of 0.2 . The underlying mica surface appears perfectly flat on the height scale used for Fig. 1a. Counting the number of clusters on several of such images results in a number density of $n=(1.9 \pm 0.2) \times 10^{9} \mathrm{~cm}^{-2}$.

b)

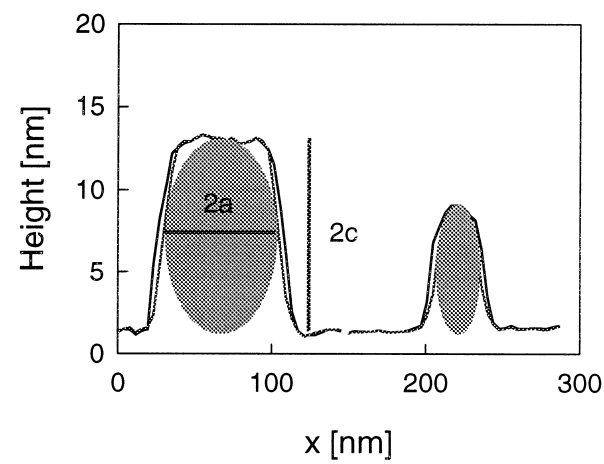

Fig. 1. (a) SFM image of Na-like clusters grown in UHV at a surface temperature of $T_{\mathrm{S}}=300 \mathrm{~K}$ on cleaved mica. The sample size is $2 \times 2$ $\mu \mathrm{m}^{2}$, the counted number density of clusters is $n=(1.9 \pm 0.2) \times 10^{9} \mathrm{~cm}^{-2}$. (b) Scan through two typical clusters on the surface. The black line shows the unprocessed scan; the grey line the result of a deconvolution with a tip of $20 \mathrm{~nm}$ diameter. Embedded spheroids used for modelling the optical properties are also shown with the two half-axes $a$ und $c$, parallel and perpendicular to the surface plane. The spheroids have symmetry axes perpendicular to the surface. 
It is well known that SFM images are a convolution of the imaged object with the imaging tip [24]. Thus, it is difficult to obtain quantitative information from these images, especially if the shape of the tip is unknown. For example, simultaneously performed TEM and SFM images of latex spheres [25] and Rutherford backscattering experiments on zinc islands [13] have shown that, in the SFM images, the nanometer-sized islands appear artefactually large. Therefore, a deconvolution of image and imaging tip has to be performed. However, even this procedure cannot reconstruct all parts of the imaged surface, due to unreconstructable regions [26], in which the tip has contacted more than one point of the imaged object at the same time, and due to imprecise modelling of the actual tip in the deconvolution algorithm. In the case of the investigated $\mathrm{Na}$ islands this implies that we cannot determine unambiguously whether the islands have the shape of (approximately) full or capped spheroids. In the past, both assumptions have been taken into account to explain the optical spectra of supported metal clusters [15,27]. In this Letter we prefer for sake of simplicity the model of full spheroids, because the spectra of flat, capped spheres in general do not differ significantly from spectra of similar oblate spheroids.

The SFM images (e.g., Fig. 1a) show flat islands of some $10 \mathrm{~nm}$ in diameter. A deconvolution [24] with a sphere of $10 \mathrm{~nm}$ radius reveals slightly smaller particles, but does not change the particle height. In Fig. 1b scans through the raw SFM image (dark lines), and scans through the deconvoluted image (grey lines) are shown. The optical properties are simulated by embedded spheroids as denoted also by shaded ellipsoidal areas in the figure. The spheroids are fitted such that their diameters perpendicular to the surface $(2 c)$ equal the heights of the islands, and the diameters parallel to the surface match the small half-axes $(2 a)$ of ellipses at half the height of the islands. Obviously, the spheroids are assumed to possess a symmetry axis perpendicular to the surface.

Fig. 2 shows measured size distributions of small and large semi-axes parallel to the surface of ellipsoids on the basis of deconvoluted clusters. In addition, the distributions of the ellipticities parallel to the surface, $R_{\|}=b / a$, and perpendicular to the surface, $R_{\perp}=c / a$, are shown. The distribution of the small half-axes (Fig. 2a) can be represented by a double-Gaussian size distribution,

$f(a,\langle a\rangle) \propto \exp \left[-\frac{1}{2}\left(\frac{a-\langle a\rangle}{\beta_{ \pm}}\right)^{2}\right]$,

with the two different widths $\beta_{+}$(for $a \geqslant\langle a\rangle$ ) and $\beta_{-}=\sqrt{2} \beta_{+}$(for $a<\langle a\rangle$ ), which account for the observed asymmetry of the distribution. The distribution of the large half-axes (Fig. 2b) and of the ellipticities (Fig. 2c,d) are best described by log-normal distributions, e.g.,

$$
\begin{aligned}
& f\left(R_{\perp},\left\langle R_{\perp}\right\rangle\right) \\
& \quad \propto \exp \left[-\frac{1}{2}\left(\frac{\ln \left(R_{\perp}\right)-\ln \left(\left\langle R_{\perp}\right\rangle\right)}{\beta}\right)^{2}\right],
\end{aligned}
$$

with the single width $\beta$. The full widths at half maximum (FWHM) of the distributions are related to the widths $\beta_{+}$and $\beta$ via

$\mathrm{FWHM}=[\sqrt{2 \ln 2}(1+\sqrt{2})] \beta_{+}$

for the double-Gaussian distribution, and via

$\mathrm{FWHM}=\exp (\beta \sqrt{2 \ln 2})-\exp (-\beta \sqrt{2 \ln 2})$,

for the log-normal distribution. The fit-curves are shown as solid lines in Fig. 2. For the distribution of small half-axes the mean value is $\langle a\rangle=35.5 \mathrm{~nm}$ with an FWHM of $85 \%$ of $\langle a\rangle$, whereas the mean ellipticity perpendicular to the surface is $\left\langle R_{\perp}\right\rangle=$ 0.18 with an FWHM of $60 \%$ of $\left\langle R_{\perp}\right\rangle$. Due to the fitting process the error for the half-axes is $\pm 5 \mathrm{~nm}$, and \pm 0.05 for the ellipticities. The absolute value of mean ellipticity is in nice agreement with that found for large (30 nm radius) silver clusters adsorbed on quartz by means of transmission electron microscopy [28].

The distribution of the large half-axes is centered around the mean value $\langle b\rangle=39 \mathrm{~nm}$ with an FWHM of $120 \%$ of $\langle b\rangle$, and for the ellipticity parallel to the surface plane one finds $\left\langle R_{\|}\right\rangle=1.2$.

A closer look at the cluster morphology using the SFM shows that the width of the distribution for the large half-axes was dictated by coagulated clusters. Therefore this information was not used for the calculation of the expected optical extinction spectra. 

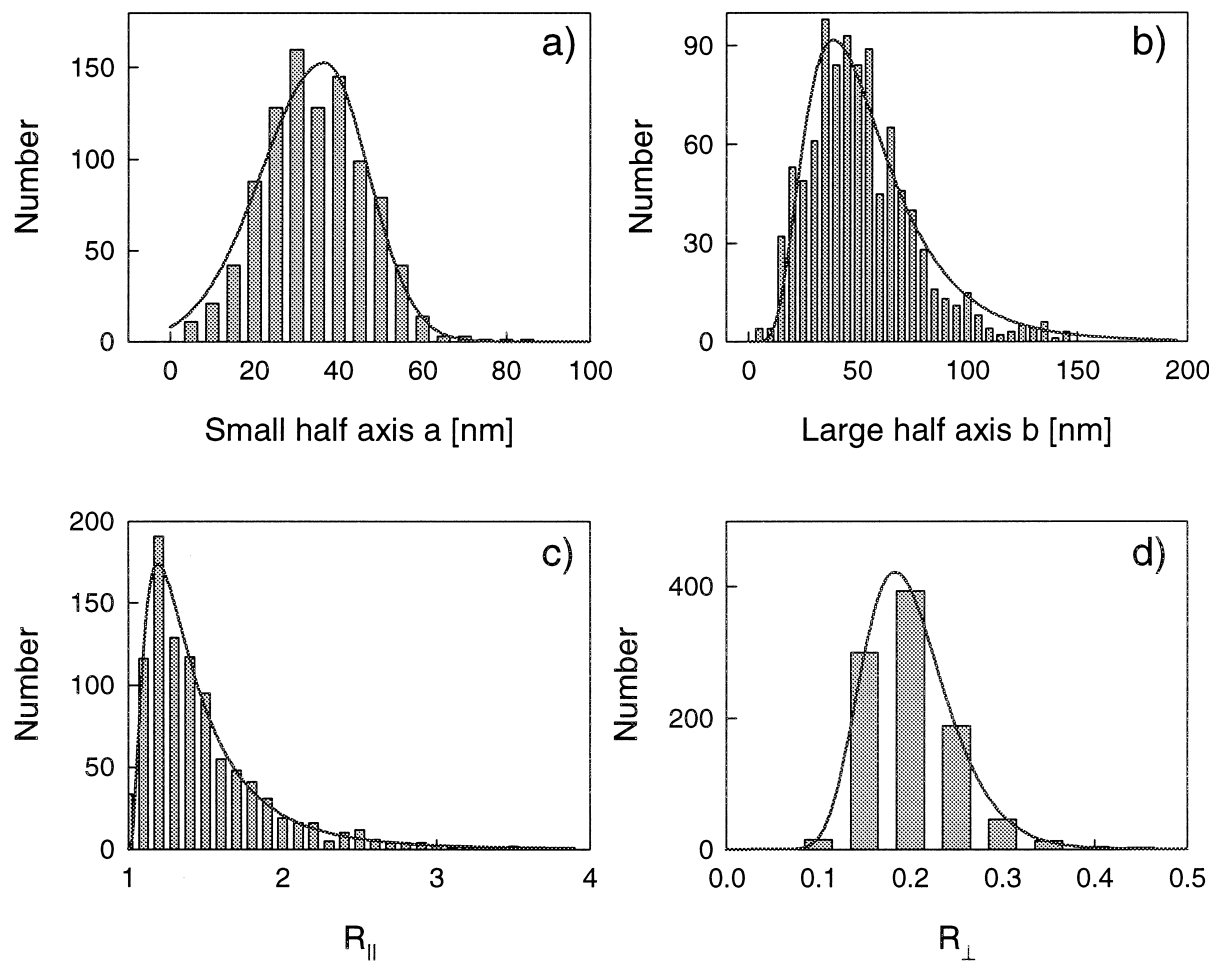

Fig. 2. Size distributions of the small (a) and large (b) half-axes parallel to the mica surface, distribution of ellipticities parallel, $R_{\|}$(c), and perpendicular, $R_{\perp}(\mathrm{d})$, to the mica surface. The solid lines are fits to the measured distributions, assuming a double-Gaussian or a log-normal distribution. For details see text.

In contrast to previous interpretations of alkali cluster distributions on surfaces on the basis of linear extinction measurements, the SFM (and TEM [11]) images suggest that the cluster size distribution cannot be described by a single value of ellipticity. The corresponding distribution of ellipticities has a severe influence on the interpretation of the extinction spectra, especially if they result from strongly oblate islands (see below).

The evolution of extinction spectra under normal incidence as a function of $\mathrm{Na}$ evaporation is shown in Fig. 3a. A prominent peak at $720 \mathrm{~nm}$ arises as a function of $\mathrm{Na}$ coverage, representing the dipole plasmon excitation of the clusters. More extensive measurements have shown [29] that the cluster morphology is sensitive to the microscopic structure of the mica surface, i.e., its preparation by cleaving and annealing. The shift to smaller wavelengths with increasing coverage is probably due to slight changes in the size and shape distributions and in the cluster density during the initial nucleation and growth process. However, without further structural information a detailed picture of the growth process remains speculative.

The final extinction spectrum $E_{\text {exp }}(\lambda)$, which led to the SFM image Fig. 1a, is shown in Fig. 3b. A calculated extinction spectrum $E_{\text {calc }}(\lambda)$, using the distribution functions for $R_{\perp}$ and $a$ from Fig. 2b, and the dielectric function of bulk $\mathrm{Na}$ [30], is also presented (grey line in Fig. 3b). The measured extinction spectrum was quantitatively and accurately reproduced. Note, that the position of the maximum for these strongly oblate islands is extremely sensitive to mean cluster size, ellipticity and the width of the distributions. The applied number density of clusters is the measured value except for a shift of the axis for the calculated spectrum by a value of 0.045, which takes into account extinction due to sodium adsorbed at the backside of the sample. Backside adsorption cannot be distinguished from 

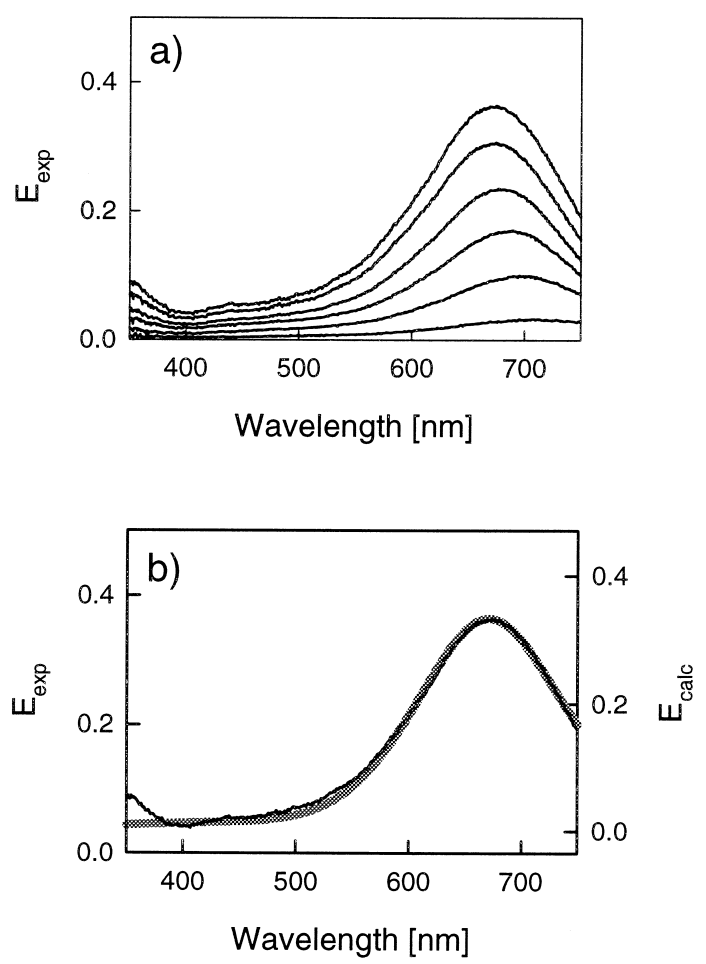

Fig. 3. (a) Measured extinction spectra during evaporation of $\mathrm{Na}$ atoms on mica at room temperature. The spectra were recorded at $16 \%, 31 \%, 47 \%, 63 \%, 83 \%$ and $100 \%$ of the final coverage. (b) Measured (black line) and calculated (grey line) extinction spectra for Na clusters adsorbed on mica. The parameters for the calculation have been taken from the deconvoluted SFM images.

frontside adsorption using linear extinction measurements. However, angular-dependent second-harmonic generation is a useful tool for obtaining this additional information [29], since it takes advantage of the interferences between the two non-linear optical sources.

The calculation of the extinction spectrum was performed by applying classical electrodynamics (T-matrix theory) $[18,29]$ and neglecting interactions between the clusters because their average distance $(240 \mathrm{~nm})$ was significantly larger than their average radius $(\approx 40 \mathrm{~nm})$. We also have assumed independent distributions of $a$ and $R_{\perp}$. In Fig. 4 extinction spectra for the same sample as in Fig. 3 are plotted for an angle of incidence of $\phi=40^{\circ}$, using s- and p-polarized light. The grey lines represent the calculated spectra, including errors due to the uncertainty in angle of incidence $\left(\Delta \phi= \pm 5^{\circ}\right)$, and the black lines are the measured spectra. In addition to using the $\mathbf{T}$-matrix formalism for non-normal incidence, the calculated spectra were scaled by a factor of $1 / \cos \phi$, thus taking into account the increasing number of probed clusters with increasing angle, assuming that the extinction cross sections of the clusters do not overlap. The calculated value of extinction for the dipole excitation parallel to the surface becomes smaller for p-polarized light because the extinction cross section decreases with $\cos ^{2} \phi$, whereas the number density increases only with $1 / \cos \phi$. This results in a net decrease of the absolute value of extinction with $\cos \phi$. For spolarized light the extinction cross section is independent of $\phi$, so that the value of extinction increases with $1 / \cos \phi$. The overall agreement between $\mathbf{T}$-matrix calculations and measured extinction spectra was very satisfactory. The slight disagreement between absolute heights of the maxima is attributed to the divergence of the light of the extinction lamp, which is difficult to account for on a quantitative basis.

Finally, we note that the use of an air-SFM to evaluate the cluster size distribution has a severe drawback. Sodium reacts with air, and therefore extinction spectra of sodium clusters, which were exposed for a few seconds to air, no longer show the characteristic extinction of a metallic sample, but appear to be nearly transparent over the whole investigated wavelength range. Hence the presented

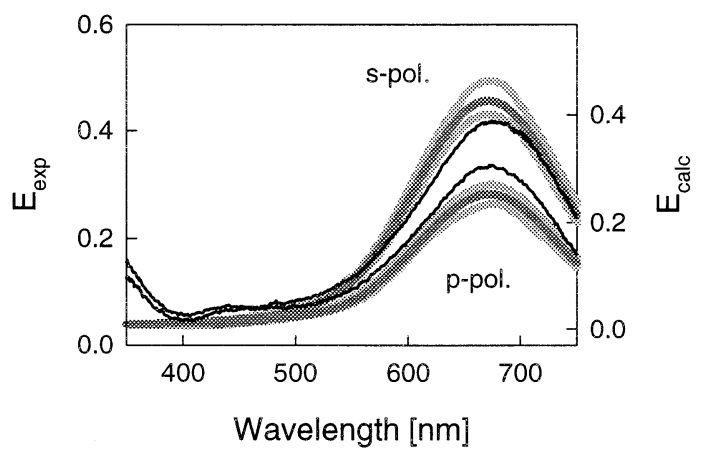

Fig. 4. Measured and calculated extinction spectra for an angle of incidence of $\phi=40^{\circ}$ for s- and p-polarized light. The parameters for the T-matrix calculations are the same as in Fig. 3b. The light grey lines show calculated spectra for $\phi=45^{\circ}$ and $\phi=35^{\circ}$, which take care of the experimental error in angle of incidence. 
SFM-image (Fig. 1) is an image of Na-like clusters, which probably consist of $\mathrm{NaOH}$. SFM images of samples one week in contact with air showed that the height of the clusters decreased further down to a few $\AA$ as a function of time. However, besides this gradual change of height, the SFM pictures also revealed that the average size and also the number density and the in-plane ellipticity of the clusters were not a function of ambient air exposure time. A similar conclusion has been obtained from previous scanning electron microscopy measurements on potassium clusters, adsorbed on $\mathrm{Si}(111)$ [31]. What remains questionable is the absolute value of the ellipticity $R_{\perp}$, which should be affected by the gradual height change due to the chemical reaction. The demonstrated agreement between measured and calculated extinction curves (Fig. 3) gives us some confidence that the initial reaction of $\mathrm{Na}$ to $\mathrm{NaOH}$ upon exposure to ambient air largely conserves the primordial morphology of the clusters. Indeed, taking the effective increase in density and mass into account that accompanies $\mathrm{NaOH}$ formation from $\mathrm{Na}$, one finds that the mean radius of the $\mathrm{NaOH}$ clusters is less than $8 \%$ smaller, compared to the mean radius of the initial $\mathrm{Na}$ clusters. Given the uncertainties imposed by the SFM tip, this effect might be safely neglected.

\section{Conclusions}

In this paper the linear optical properties of large, flat sodium clusters, grown on mica were investigated and theoretically reproduced by using morphological information from SFM images. It appears that for our system interactions of the clusters with the substrate can be safely neglected as compared to the huge influence of the distribution of cluster ellipticities on the observed extinction spectra. The present method is to be compared with a widespread indirect approach represented in the literature to deduce alkali cluster size distributions from measured extinction spectra. This approach implements Mie theory for spherical particles and introduces a certain value of ellipticity of the clusters by using an effective dielectric function [32]. Another method is to neglect retardation effects and to calculate the spectra in the regime of the unshifted dipole resonance for small spheroids [28], or to introduce cluster-substrate [33] and cluster-cluster dipole interactions [34]. Also, for a quantitative comparison between measured and calculated extinction spectra one usually introduces a cluster density which equals the defect density of the supporting surface, which itself is a more or less arbitrary value.

The results of most of these theoretical methods and especially the simple Mie theory, applied to the extinction spectrum of Fig. 3, would disagree with our findings and with the SFM data. However, even if one uses the more accurate $\mathbf{T}$-matrix theory on a size distribution of clusters with an FWHM of $50 \%$ of $\langle a\rangle$ and no distribution of ellipticities, one finds as a best fit a significantly larger value of small semi-axis, namely $\langle a\rangle=84 \mathrm{~nm}, R_{\perp}=0.3$ and a value of cluster density which clearly is too small, namely $n=2.5 \times 10^{8} \mathrm{~cm}^{-2}$. It is not possible to trace these discrepancies back to reactions of the $\mathrm{Na}$ clusters with ambient air since those reactions do not affect significantly the number density of the clusters. In addition, those large clusters would be clearly visible in the extinction spectra for finite angles of incidence due to dipole excitations perpendicular to the substrate surface, which was obviously not the case (cf., Fig. 4). In the context of such approximate calculations the broadening of the spectrum would be attributed to a majority of larger particles, not to the distribution of ellipticities. The present work suggests that the latter factor has the main influence on the extinction spectra. Obviously, such an effect becomes most important for a distribution of flat particles. For a distribution of more spherical particles the broadening due to the different shapes is not severe as will be shown in a future publication on the spectra of alkali clusters bound to organic films.

It clearly would be very useful to apply the method developed in the present paper to data taken with an UHV-SFM that avoids problems with reactions of the $\mathrm{Na}$ clusters. One also should vary the supporting surface and make sure that its influence via image dipole and multipole interactions is negligible in the case of sodium clusters on mica (in contrast to, e.g., silver clusters on silicon substrates [28]). A possible alternative would be to cover the clusters with a shield of organic molecules and in that way avoid reactions with the ambient air. Such studies are underway in our laboratory. 


\section{Acknowledgements}

We are grateful to J.P. Toennies for his continuous support and to T.M. Jovin for critical reading of the manuscript.

\section{References}

[1] J. Chen, D.M. Brink, L.T. Wille, J. Phys. B 23 (1990) 885.

[2] U. Kreibig, M. Vollmer, Optical Properties of Metal Clusters, Springer Series in Materials Science, vol. 25, Springer, Berlin, 1995.

[3] Th. Müller, P.H. Vaccaro, F. Balzer, H.-G. Rubahn, Optics Comm. 135 (1997) 103.

[4] F. Balzer, H.-G. Rubahn, Chem. Phys. Lett. 238 (1995) 77.

[5] J.-H. Klein-Wiele, P. Simon, H.-G. Rubahn, Phys. Rev. Lett. 80 (1998) 45.

[6] H.-G. Rubahn, Appl. Surf. Sci. 109/110 (1997) 575.

[7] H.-G. Rubahn, Trends Chem. Phys. 6 (1997) 31.

[8] R. Gerlach, J.R. Manson, H.-G. Rubahn, Optics Lett. 21 (1996) 1183.

[9] K. Reichelt, Vacuum 38 (1988) 1083.

[10] S. Norrman, T. Andersson, C.G. Granqvist, Phys. Rev. B 18 (1978) 674.

[11] M. Rasigni, G. Rasigni, J.P. Gasparini, R. Fraisse, J. Appl. Phys. 47 (1976) 1757.

[12] J.-D. Grunwaldt, F. Atamny, U. Göbel, A. Baiker, Appl. Surf. Sci. 99 (1996) 353.

[13] M.F. Tabet, F.K. Urban III, J. Vac. Sci. Technol. B 15 (1997) 800.

[14] H. Schmeisser, Thin Solid Films 22 (1974) 83.
[15] W. Hoheisel, U. Schulte, M. Vollmer, F. Träger, Appl. Phys. A 51 (1990) 271.

[16] G. Mie, Ann. Phys. Leipzig 25 (1908) 377.

[17] P.C. Waterman, Phys. Rev. D 3 (1971) 825.

[18] M.I. Mishchenko, L.D. Travis, D.W. Mackowski, J. Quant. Spectrosc. Radiat. Transfer 55 (1996) 535.

[19] S.E. Roark, K.L. Rowlen Chem. Phys. Lett. 212 (1993) 50.

[20] E. Palange, L. Ragne, L. Di Gaspare, G. Capellini, F. Evangelisti, J. Appl. Phys. 83 (1998) 5840.

[21] R. Gerlach, H.-G. Rubahn, to be submitted.

[22] M. Ohring, The Materials Science of Thin Films, Academic Press, San Diego, CA, 1992.

[23] C. F Bohren, D.R. Huffman, Absorption and Scattering of Light by Small Particles, John Wiley, New York, 1983.

[24] P. Markiewicz, M.C. Goh, Langmuir 10 (1994) 5.

[25] K.A. Ramirez-Aguilar, K.L. Rowlen, Langmuir 14 (1998) 2562.

[26] E.C.W. Leung, P. Markiewicz, M.C. Goh, J. Vac. Sci. Technol. B 15 (1997) 181.

[27] T. Yamaguchi, H. Takahashi, Surf. Sci. 78 (1978) 295.

[28] P. Royer, J.L. Bijeon, J.P. Goudonnet, T. Inagaki, E.T. Arakawa, Surf. Sci. 217 (1989) 384.

[29] F. Balzer, Ph.D. Thesis, University of Göttingen, 1998.

[30] E.D. Palik, editor, Handbook of Optical Constants of Solids. Academic Press, Orlando, FL, 1985.

[31] B. Lamontagne, F. Semond, A. Adnot, D. Guay, D. Roy, Appl. Phys. A 61 (1995) 187.

[32] T. Götz, W. Hoheisel, M. Vollmer, F. Träger, Z. Phys. D 33 (1995) 133.

[33] U. Kreibig, M. Gartz, A. Hilger, Ber. Bunsenges. Phys. Chem. 101 (1997) 1.

[34] R.R. Singer, A. Leitner, F.R. Aussenegg, J. Opt. Soc. Am. B 12 (1995) 220 